\title{
Growth Stages of Nano-structured Mixed-phase Titania Thin Films and Effect on Photocatalytic Activity
}

\author{
Aleksandra J. Gardecka ${ }^{a}$, Matthew I. J. Polson ${ }^{b}$, Susan P. Krumdiecka ${ }^{a}$ Yicun Huang ${ }^{a}$, \\ Catherine M. Bishop ${ }^{\mathrm{a}, *}$ \\ ${ }^{a}$ Department of Mechanical Engineering, University of Canterbury, Private Bag 4800, Christchurch, \\ New Zealand \\ ${ }^{b}$ School of Physical and Chemical Sciences, University of Canterbury, Private Bag 4800, Christchurch, \\ New Zealand
}

\begin{abstract}
The development of nano-structured mixed-phase titania thin films and the relationship to photocatalytic activity (PCA) is of interest for pollution reduction and antimicrobial applications. A set of films grown by pulsed pressure metallorganic chemical vapour deposition using titanium tetraisopropoxide precursor was studied. The growth method is single-stage, scalable and produces high-quality, adherent films. Film thicknesses were $101 \mathrm{~nm}$ to $4.0 \mu \mathrm{m}$. Three growth stages were identified. All films were a mixture of anatase and rutile. Early stage films had rounded morphologies, low surface roughness and low PCA. Transition stage films developed columnar [110] orientated anatase dendrites, contained amorphous carbon and had reduced UV transmittance. Late stage films had strong anatase (220) and rutile (200) textures, contained amorphous carbon and exhibited highly branched anatase dendrites with nanoscale secondary plates. PCA was determined from degradation of stearic acid and correlated with increasing surface roughness. The most active film had formal quantum efficiency of $(6.62 \pm 0.64) \times 10^{-4}$ molecules/photon, 59 times higher than a commercially available control. The performance is attributed to the combination of phases yielding polymorphic phase boundaries and amorphous carbon enhancing the adsorption of organic molecules, the unusual (220) anatase texture yielding nanostructured anatase dendrites in combination with nanocrystalline rutile and hierarchical porosity.
\end{abstract}

Keywords: titania, pulsed pressure metal-organic chemical vapour deposition, film development, photocatalysis, surface roughness, hierarchical porosity

\footnotetext{
*Corresponding author

Email address: catherine.bishop@canterbury.ac.nz (Catherine M. Bishop)
} 


\section{Introduction}

Titanium dioxide thin films, both in pristine and variously doped forms, play significant roles in many areas of chemistry, physics, biology and material science, where they find applications in catalysis, photonics, optics, electronics, window coatings, antimicrobial coatings for touch surfaces and energy storage/conversion devices [1 5]. Thin films exhibit different physiochemical properties depending on the method of deposition, particle size, film density, level of crystallinity and nanostructural geometry. Crystallite size and shape, as well as the film's surface area, play crucial roles in the catalytic and electrochemical behaviour [6].

As reported extensively in the literature, nanostructured thin films with secondary and tertiary structures oriented in radial or axial directions from a primary backbone exhibit superior electrochemical performances compared to monolithic materials [7]. Additionally, it is well known that mixed phase, titania photocatalysts containing anatase and rutile perform better than single phase materials due to favourable band alignment [8, 9]. These heterostructures have been tailored by fine-tuning the size, shape and composition to enhance material properties. Metal-organic chemical vapour deposition (MOCVD) is often used in synthesis of nanostructured thin films, as it allows control of phase fractions and microstructures simply by regulation of the temperature of the deposition process and precursor arrival rate, as well as length of the deposition [2, 10, 11]

In this paper we use the pulsed-pressure MOCVD (pp-MOCVD) method to produce titania thin films from a single liquid-source precursor solution of titanium tetraisopropoxide (TTIP) in toluene. The pp-MOCVD method is scalable, produces conformal films on a variety of substrates and allows control of the microstructure of films by changing deposition conditions [12]. Previous work on microstructural development of pp-MOCVD titania films has focused on the effect of processing parameters including substrate temperature and dose per pulse on mature films [13, 14]. Functional performance of pp-MOCVD films has been evaluated in two studies: water splitting efficiency was studied for a series of titania films grown at a single temperature on FTO (fluorine doped tin oxide) -coated glass[2], and antimicrobial activity was evaluated for a titania film on stainless steel[15].

We identify (220) textured anatase dendrites in thicker films in this work. There are a few reports of (220) textured anatase in the literature grown by other CVD (chemical vapour deposition) methods using TTIP precursor, but photocatalytic activity (PCA) 
has not been evaluated for those materials. Takahashi et al. reported the growth of (220) textured anatase on glass substrates by conventional CVD using TTIP and oxygen with nitrogen carrier gas at $500{ }^{\circ} \mathrm{C}[10]$. Higher flow rates and longer times favoured the formation of rutile, which dominated the upper surface in films greater than $10 \mu \mathrm{m}$ thick. Due to the strong anatase texture, they noted that phase fractions from x-ray diffraction (XRD) were not reliable. No nanostructure or mention of carbon was reported. Growth rates in steady state of $250 \mathrm{~nm} \mathrm{~min}^{-1}$ were indicated.

Seifering et al. reported (220) textured anatase grown on copper from TTIP with no carrier gas[16]. They stated that XRD showed that the films were amorphous or microcrystalline, then (220) textured anatase and then rutile as thickness increased. But no XRD patterns or images of the films were provided for comparison. No nanostructure or mention of carbon was reported. The growth rate at $300^{\circ} \mathrm{C}$ was $167 \mathrm{~nm} \mathrm{~min}^{-1}$.

Chen et al. reported (220) textured anatase nanocrystalline films of $5 \mu \mathrm{m}$ thickness obtained on fused silica in low pressure chemical vapour deposition (LPCVD) at $550{ }^{\circ} \mathrm{C}$ and $150 \mathrm{~Pa}$ total pressure using TTIP with oxygen carrier gas[17]. Adventitious carbon was found in x-ray photospectroscopy (XPS). The C1s peak persisted after ion milling, but no explanation was given. No growth rate or growth time was reported. The authors also showed that rutile could be produced at higher pressures, $150 \mathrm{~Pa}-500 \mathrm{~Pa}$, under the same conditions on a sapphire substrate[18].

The development of microstructure and properties of pp-MOCVD films with increasing deposition time has not previously been studied. Because deposition in done in pulses, this provides the interesting ability to explore the stages of film evolution, which is exploited here. In this study, we explore the development of nano- and microstructure in a series of samples with different deposition times, and determine the impact of film structure on the development of photocatalytic performance by degradation of stearic acid.

\section{Experimental Details}

\subsection{Synthesis of $\mathrm{TiO}_{2}$ thin films}

A set of $\mathrm{TiO}_{2}$ films was grown on fused silica substrates $(25 \mathrm{~mm} \times 25 \mathrm{~mm} \times 1 \mathrm{~mm})$ using pp-MOCVD in a cold wall reactor, previously described by Lee et al. [19]. Heating was by induction coil with deposition temperature measured by a k-type thermocouple 
inserted into the susceptor. The substrate temperature was maintained at $525^{\circ} \mathrm{C} \pm 5{ }^{\circ} \mathrm{C}$. A metered volume of $500 \mu \mathrm{L}$ of precursor liquid solution (5 mol\% TTIP in toluene) was directly injected through an ultrasonic atomising nozzle into the continuously evacuated reactor volume at timed intervals, resulting in a sharp pressure pulse followed by pumpdown. The injection pulses were spaced $6 \mathrm{~s}$ apart. The chamber base pressure was held at $120 \mathrm{~Pa}$, with the mean peak pressure in the region of $500 \mathrm{~Pa}$. In the current reactor geometry, the susceptor was placed directly above an induction coil, and the injector was located at the top of the reactor. The samples were prepared with different numbers of pulses from 10 to 300 in order to investigate evolution of structure and properties.

\subsection{Materials characterisation}

XRD was used for phase identification and texture analysis. This was carried out on an Agilent SuperNova, Dual, $\mathrm{Cu}$ at zero, Atlas diffractometer using $\mathrm{Cu} \mathrm{K}_{\alpha}(\lambda=1.5418 \AA)$ radiation. Samples were fixed with the surface at an angle $\omega \sim 15^{\circ}$ to expose a large region of the surface to the $0.3 \mathrm{~mm}$ diameter beam. Post collection, the frame was integrated over a $5^{\circ}$ strip corresponding to a pseudo $\theta-2 \theta$ diffractometer. To correct for slight differences in the mounting alignment, the angle in $\gamma$ that this $5^{\circ}$ strip was integrated over was calculated as the average of two off-axis, symmetry-related peaks at $2 \theta=25^{\circ} .2 \theta$ angles were corrected to P25, and the baselines were manually removed for later texture analysis.

Film thickness and surface morphology were observed by scanning electron microscopy (SEM) using a JEOL 7000F Field Emission SEM at accelerating voltage of $15 \mathrm{keV}$ on Cr-coated samples. Film morphology was further studied using a Philips CM 200 transmission electron microscope (TEM) and a JEM-2200FS scanning transmission electron microscope. Specimens were prepared by removing a portion of film from the substrate using a diamond blade, applying it onto a $\mathrm{Cu}$ grid and coating with carbon. Results were processed using CrystBox software[20].

Surface topography was characterised using a Digital Instruments Dimension 3100 atomic force microscope (AFM) operating in tapping mode with a scan frequency of $2 \mathrm{~Hz}$ over a $500 \mathrm{~nm} \times 500 \mathrm{~nm}$ area. Results were processed using Gwyddion software[21].

Transmittance spectra were taken against an air background using a Cintra $404 \mathrm{UV}$ Vis spectrometer over a wavelength range of $200 \mathrm{~nm}-2500 \mathrm{~nm}$. The double monochromator spectrometer was used with a slit width of $2.0 \mathrm{~nm}$. 
A Jobin-Yvon LabRam single stage Raman spectrometer with $514.5 \mathrm{~nm}$ Ar-ion laser (420 $\mu \mathrm{W}$ power, $10 \mu \mathrm{m}$ beam diameter) was used to characterise the films.

\subsection{Photocatalytic testing}

The photocatalytic performance of the films synthesised in this work was evaluated by observing photodegradation of octadecanoic (stearic) acid (95\%, Sigma-Aldrich). Films were dip-coated with a thin layer of stearic acid using a $0.05 \mathrm{M}$ solution in chloroform. The PCA of samples was measured under UVA (Vilber-Lourmat, $2 \times 8 \mathrm{~W}, 365 / 254 \mathrm{~nm}$, $0.4 \mathrm{~mW} \mathrm{~cm}^{-2}$ ) illumination. A Bruker Vertex 70 Fourier transform infrared spectrometer was used to monitor the degradation of the overlain stearic acid.

The integrated areas between the characteristic $\mathrm{C}-\mathrm{H}$ infrared bands at $2958 \mathrm{~cm}^{-1}$, $2923 \mathrm{~cm}^{-1}$ and $2853 \mathrm{~cm}^{-1}$ were calculated. The photoactivity rates were estimated from linear regression of the initial $30 \%-40 \%$ of the degradation, where the reaction has been shown to possess zero-order reaction kinetics above a critical stearic acid concentration. Using a conversion factor, the number of molecules of stearic acid degraded was estimated considering that $1 \mathrm{~cm}^{-1}$ of integrated area corresponds to $9.7 \times 10^{15}$ molecules of stearic acid[22]. Formal quantum efficiencies (FQE), defined as molecules of acid degraded per incident photon (molecules/photon), were calculated assuming all incident photons had the same energy of $3.4 \mathrm{eV}$ and were absorbed by the films. Three tests were performed per sample and the mean PCA reported.

\section{Results}

\subsection{Morphology}

Continuous films were observed on all substrates, Fig. 1. Three stages of growth were identified. The morphology of the films began with rough-looking, rounded structures with linear cross-sectional dimensions less than $100 \mathrm{~nm}$ in the early stages (10 pulses40 pulses). In the transition stage, separated, columnar structures with linear dimensions greater than $100 \mathrm{~nm}$ were observed (70 pulses-80 pulses). Eventually, segmented, prismatic-topped, large columns with linear dimensions of $\sim 400 \mathrm{~nm}$ in the thickest sample studied here (300 pulse) formed in late stage growth (90 pulses-300 pulses).

Cross-sections and AFM topographic maps of early stage and late stage films illustrate the stages of film development, Fig. 2. In early stage growth, the films were composed of 
rough, near-spherical clusters of material with maximum height differences on the order of $20 \mathrm{~nm}$, Fig. 2(a) and (c). In late stage growth, the films were clearly columnar with secondary branching and tertiary arms visible in Fig. 2(b). The secondary plates on the prismatic tops of the large columns in the 300 pulse sample were evident in AFM scan, which also showed the maximum height difference was on the order of $300 \mathrm{~nm}$, Fig. 2(d). The secondary plates were visible in the transition stages as illustrated for 80 pulse sample in Fig. 2(e).

The thickness of each film was determined from the cross-sectional SEM images by averaging five measurements, Table 1. The thickness increased linearly with number of pulses with a rate of $4 \mathrm{~nm} /$ pulse $\left(37 \mathrm{~nm} \mathrm{~min}^{-1}\right)$ during early stage growth $(\leq 40$ pulses where $R^{2}>0.99$ ), and became non-linear during the transition stage, and accelerated for the thicker films in late stage growth, Fig. 3(a). Average growth rates (total film thickness divided by the total deposition time) as a function of deposition temperature are commonly reported in the literature to distinguish the CVD growth regimes of mass transport control from kinetic control. The average growth rate for the thickest sample here was $130 \mathrm{~nm} \mathrm{~min}{ }^{-1}(13 \mathrm{~nm} /$ pulse $)$, twice the initial linear rate.

The surface area ratio (total surface area divided by projected surface area from AFM) and the RMS roughness were determined from AFM data, Table 1 . In early stage growth, the RMS (route mean square) roughness was less than $5 \mathrm{~nm}$. Roughness approximately doubled in transition stage compared to early stage films. Finally, RMS roughness in late stage films was up to $61 \mathrm{~nm}$. The area ratio did not show such a dramatic change, but did increase with film growth. The area ratio plateaued in late stage growth while the RMS roughness continued to increase for the three thickest samples: from 120 pulses $(0.7 \mu \mathrm{m}$ thick) to 300 pulses ( $4.0 \mu \mathrm{m}$ thick), the RMS roughness tripled from $18 \mathrm{~nm}-62 \mathrm{~nm}$ while the area ratio was unchanged, at 1.7, Fig. 3(b). As shown in previous studies, surface area and RMS roughness of CVD grown films increase with film thickness, while those properties are independent of film thickness in sol-gel grown films[23].

\subsection{Phase identification}

XRD revealed that all the films were mixed-phase titania with anatase and rutile detected in every sample, Fig. 4. No other crystalline phases were identified. Both phases were textured and as a result the phase fractions and average diffracting crystallite size could not be determined from these measurements. 
Texture Coefficients (TC) for each phase were calculated separately[24],

$$
\mathrm{TC}(\mathrm{hkl})_{i}=\frac{\frac{I(h k l)_{i}}{I_{0}(h k l)_{i}}}{\frac{1}{N} \sum_{i}^{N} \frac{I(h k l)_{i}}{I_{0}(h k l)_{i}}}
$$

where $\mathrm{I}(\mathrm{hkl})_{\mathrm{i}}$ is the observed intensity of the $(\mathrm{hkl})_{\mathrm{i}}$ reflection and $\mathrm{I}_{0}(\mathrm{hkl})_{\mathrm{i}}$ is the intensity of the $(\mathrm{hkl})_{\mathrm{i}}$ reflection of a polycrystalline reference sample of the phase of interest. $\mathrm{N}$ is the total number of reflections used in the analysis. A reference sample of P25 was collected on the same instrument to allow direct comparison, and gave similar intensities to the literature values 25$]$. Five reflections $(\mathrm{N}=5)$ were included in the TC for anatase: approximate $2 \theta(\mathrm{hkl})=25^{\circ}(101), 48^{\circ}(200), 55^{\circ}(211), 70^{\circ}(220)$ and $74^{\circ}(215)$. Three reflections $(\mathrm{N}=3)$ were included in the TC for rutile: $27^{\circ}(110), 36^{\circ}(101)$ and $39^{\circ}(200)$.

Both phases developed strong texture in the thicker films: (220) texture in anatase and (200) texture in rutile, Fig. 3(c). Error analysis on the anatase XRD data gave relative errors of less than $5 \%$ on TC for transition and late stage films. The rutile texture reached a maximum $\mathrm{TC}(200)=3.0$ in a transition stage film, while the anatase texture reached its maximum of $\mathrm{TC}(220)=4.5$ in the thickest film. This (220) anatase texture is consistent with previous pure anatase films grown in the same reactor on fused silica substrates at lower temperatures $\left(375^{\circ} \mathrm{C}-450^{\circ} \mathrm{C}\right)$, but the texture in the lower temperature materials was not as strong[14].

Raman spectra were analysed for evidence of carbon, Fig. 5. The Raman shift in the range $1360 \mathrm{~cm}^{-1}-1650 \mathrm{~cm}^{-1}$ is attributed to $\mathrm{D}$ and $\mathrm{G}$ peaks for carbon, typical for MOCVD, and is identified as amorphous carbon[26]. Amorphous carbon is evident in the transition and late stage samples with thicknesses greater than $320 \mathrm{~nm}$, but not in the first three early stage samples. No graphite or diamond phases were identified in the XRD suggesting that the structured carbon has a low volume fraction or is absent. This is consistent with XPS analysis of titania films deposited under the same conditions on FTO-coated glass substrates in previous work, where we identified amorphous carbon throughout the depth in addition to the expected, adventitious carbon on the top surface of the films[2]. The improved water splitting efficiency after annealing supported the conclusion that the amorphous carbon had formed surficial layers at interdendritic and interphase interfaces in those mixed-phase titania films. 


\subsection{Structure}

TEM was used to investigate portions of films removed from the substrates, Fig. 6. The 20 pulse $(\sim 140 \mathrm{~nm})$ sample was composed of equiaxed clusters of material on the order of $100 \mathrm{~nm}$ in dimension, Fig. 6(a). For longer deposition time, clusters of approximately the same size were evident on the TEM grid, Fig. 6(b), as well as continuous, amorphous layers, not shown. These are characteristic of early stage growth. The rodlike, nano-structured dendritic nature of the fully-developed, late stage growth films is illustrated for the 200 pulse $(1.1 \mu \mathrm{m})$ sample, Fig. 6(c)-(e). Fig. 6(c) contains two anatase dendrites rotated approximately $90^{\circ}$ to each other about the growth direction. The collection of secondary plates that branch from the primary stem are edge-on in the left-hand dendrite. The right-hand dendrite is orientated so that the plates are approximately in-plane, and tertiary dendritic branches are visible. The width of the anatase secondary plates and the tertiary branches was $10 \mathrm{~nm}-20 \mathrm{~nm}$. Selected area electron diffraction (SAED) analysis of the yellow circled region of a dendrite from Fig. 6(d) is shown in Fig. 6(e). The primary growth direction was identified as anatase [110], consistent with the anatase (220) texture identified in XRD.

Fully-formed dendrites as in Fig. 6(c), reported previously by us to be nanostructured anatase single crystals grown under similar condition on FTO-coated glass substrates, were directly observed in the transition and late stage 80 pulse- 300 pulse samples by SEM, but not for shorter deposition times[2]. The only crystalline phase identified in TEM samples was anatase. No rutile was identified here by TEM analysis, even though it was detected in XRD, Fig. 4, suggesting that those crystallites must be small and interspersed between the larger anatase dendrites or were preferentially destroyed in the TEM sample preparation. No carbon structures were found in the TEM analysis suggesting that carbon formed thin layers at crystallite interfaces only[2].

\subsection{Optical properties}

The thinest films were transparent and the thickest films opaque to visual inspection. The transmittance in UV-vis gradually decreased as the films became thicker and more defined nanostructures were formed to the point where the film became almost opaque at $\sim 4 \mu \mathrm{m}$ thick, Fig. 7, consistent with Seifering[16]. This phenomenon can be related to the increase in the surface area and development of dendritic structure, increasing light trapping and scattering ability[27]. The periodic features of the spectra in the 
thinner films were attributed to the low film thicknesses. Analysis of the spectra for changes in band gap are meaningless because the films are composites of anatase, rutile and amorphous carbon. Further, the Beer-Lambert law states that the transmittance will depend on the concentrations of the phases and their optical properties, and those concentrations were unable to be determined in these samples.

\subsection{Photocatalytic activity}

The PCA of our materials were compared with a blank substrate (fused silica) and an industrially available standard, Pilkington Activ $^{\mathrm{TM}}$ [28], with FQEs determined as $(0.029 \pm 0.024) \times 10^{-4}$ molecules/photon and $(0.110 \pm 0.064) \times 10^{-4}$ molecules/photon, respectively, Fig. 8, All of the pp-MOCVD films performed as well as Activ ${ }^{\mathrm{TM}}$, with some scatter of the means and standard deviations, Table 1. Early stage and transition stage films performed about as well as each other even though the film thicknesses ranged from $101 \mathrm{~nm}-519 \mathrm{~nm}$. The best performing sample was 200 pulses $(\sim 1 \mu \mathrm{m}$ thick $)$ with FQE of $(6.62 \pm 0.64) \times 10^{-4}$ molecules/photon, a factor of 59 higher than our measurements on Activ $^{\mathrm{TM}}$. This exceeds reported FQE of $2.57 \times 10^{-4}$ molecules/photon for N-doped titania films grown by APCVD [29] and is comparable to hierarchically porous anatase films with $(7.0 \pm 0.3) \times 10^{-4}$ molecules/photon[30]. Caution should be used in directly comparison of reported FQEs because measured values increase with increasing initial loading of stearic acid and reducing UV intensity[30].

\section{Discussion}

\subsection{Structure development}

The nucleation stage dominated by substrate interactions was not investigated here as the thinnest film in this study was $101 \mathrm{~nm}$ thick. The film thickness increased linearly with time in early stage growth and accelerated in late stage growth. The film growth rates reported here are calculated from the number of pulses multiplied by the time per pulse, but the growth is in a continuously changing reactor unlike most steady state MOCVD processes. In addition, in-situ growth rate determination often employs a measured mass and an assumed density for fully-dense anatase to convert to growth rate. Caution should be used in making direct comparisons of our growth rates to literature values.

Film growth is often non-linear with time, and is observed to accelerate once a preferred orientation of columnar crystals is obtained in Zone-T film microstructures, also 
called non-equiaxed microstructures[31, 32]. Zone-T films are expected at intermediate homologous growth temperatures [31]. The obtained texture is a result of preferential growth direction, and the coarsening of the columnar grain cross-sections is a result of competitive film growth and lateral grain growth[32].

Late stage growth was also associated with highly branched anatase dendrites growing along the [110] direction. Dynamic scaling theories have been used to isolate the dominant film growth physics for various growth methods including CVD, sputtering and evaporation 33]. These use statistical measures of the surface morphology of films to determine power law scaling exponents. RMS roughness, called interface width in that field, scales with growth time $R M S \sim t^{\beta}$ with the growth exponent $\beta$. The log-log plot of RMS roughness with growth time, Fig. 9, revealed two regimes corresponding to early stage, $\beta=0.83$, and late stage growth, $\beta=1.3$. This is consistent with Almeida et al. who identified two regimes during CdTe growth with films that were untextured in early growth and developed a texture in late stage growth[34]. Theory predicts a maximum growth exponent of unity in a single phase material, and the late stage exponent here is larger than that. These scaling results should be considered preliminary as they are based on one AFM map per sample and the growth time covers fewer than two orders of magnitude.

The anatase dendrite morphology, including the nanostructure in our thicker films and [110] growth direction, are consistent with anatase grown by Chen et al.[17]. They used XRD and TEM to determine the crystallography, but the identification of the large surfaces of the secondary dendrite arms was inconsistent with their characterisation. They proposed that these were the high energy $\{001\}$ surfaces, see their Fig. 8, but these dendrite surfaces were inclined to the substrate normal which precludes this identification because $\{001\}$ planes are orthogonal to $\{220\}$ in anatase. They proposed oriented attachment as the growth mechanism, but this requires primary particle formation that is unlikely in LPCVD compared to solution-based hydrothermal growth[35].

In our samples, rutile was detected by XRD, but none was identified in TEM analysis. This suggests that the phase fraction of rutile was low in these $<5 \mu \mathrm{m}$ films. If thicker samples were grown, the phase fraction of rutile may increase after an incubation time that will likely vary with temperature, as reported by Takahashi et al. [10]. The anatase to rutile transformation has been observed to nucleate on (112) twins in 
nanocrystalline anatase 36] and has been calculated to occur after surface reconstruction of $\{112\}$ facets [37]. No anatase (112) twins were observed in our samples by TEM.

Amorphous carbon was detected by Raman spectroscopy in transition and late stage films studied here. It is expected to be present throughout the film thickness, as in our previous work[2]. The growth mechanism of the mixed anatase, rutile and carbon composite is an open question. Carbon is commonly viewed as a contaminant in MOCVD, but here we speculate that it plays a role in both the development of the film morphology and in the photocatalytic behaviour. That none was detected in the three thinnest films may be due to an autocatalytic growth effect.

\subsection{Photocatalytic activity}

The PCA of our set of films increased with increasing RMS roughness measured by AFM, Fig. 10. The FQEs of the two thickest films were $(6.62 \pm 0.64) \times 10^{-4}$ molecules/photon and $(5.84 \pm 1.09) \times 10^{-4}$ molecules/photon and correspond to the highest RMS roughnesses. Given that the hierarchical porosity extends below the top surface of the film and that there are overhangs due to the branching that are inaccessible to the AFM tip, Fig. 2(b), AFM measurements will underestimate the actual surface area available for photocatalysis. Some dynamic scaling models predict that a saturation RMS roughness will be obtained at a critical time[38]. In which case, if the RMS roughness alone determines the PCA, then PCA is expected to reach a maximum after which no further increase will be obtained. On the other hand, if thicker films contain more rutile and that phase starts to dominate the upper layer of films at longer growth times, then the PCA would be expected to decrease at longer growth times. Both suggest that an optimum growth time exists.

Jung et al. found that PCA measured with methylene blue dye increased with increasing film thickness up to approximately $5 \mu \mathrm{m}$ and then plateaued for thicker titania films grown by LPCVD at $500{ }^{\circ} \mathrm{C}[39]$. In a previous paper, XRD patterns were indexed as (112) textured anatase at that temperature, but no growth time was given[40]. Subsequent work showed that specific surface area and PCA were proportional in these films that are assumed to be phase pure anatase 23] Our results are consistent with a plateau in PCA with increasing thickness, but the relationship to the specific surface area is unknown and our materials are mixed phase.

Structures that increase light absorption, reduce the recombination of photogenerated 
carriers, increase the number of surface reaction sites and decrease the barrier for surface reactions promote $\mathrm{PCA}$.

Highly branched nanostructured materials increase light absorption compared to smooth surfaces. The early stage films are transparent and the late stage films are opaque. The opacity occurred in the most highly branched structures with multi-scale roughness.

Reduced recombination of charge carriers has been linked to internal electric fields that drive charge separation of electrons and holes. Li et al. listed polymorphic junctions and space charge regions at interfaces as sources of these fields 41]. We postulate that rutile is dispersed through the films as small crystallites in electrical contact with anatase and this allows efficient charge transfer across polymorphic junctions. While not able to be quantified, it is likely that the rutile phase fraction increases during late stage growth. The anatase crystallites possess secondary dendrite structures that are plate-like and would have fields perpendicular to the large surfaces. The exact nature of these surfaces was not determined here.

Oxidation and reduction reactions are expected to occur on the surfaces exposed to stearic acid. This is a surface phenomenon; therefore, the film thickness should not have a direct effect. This is unlike our previous water splitting experiments on related materials where electrons must be transported across a film to the electrode, and thicker films had reduced performance[2]. The surface area available for reaction will be underestimated by AFM measurements due to the morphology, but cannot be measured on films using the common nanoparticle methods like BET[42].

The adsorption of organic molecules is promoted in carbonaceous titania composites compared to pure titania, and we postulate that the surficial, amorphous carbon here serves that function[43]. While the amount of carbon could not be quantified, it was not evident in the thinnest films and its lack may have contributed to their low PCA.

The performance of the two thickest samples was significantly better than the thinner samples here and coincided with the development of the highly branched, aligned anatase dendrites with micro and nano porosity. There are a few reported multi-step methods to synthesise titania films (rather than immobilised nanoparticles) with hierarchical porosity, such as templated sol-gel methods or acid leaching of aerosol assisted CVD grown mixtures of zinc oxide and zinc titanate[30, 44]. In contrast to these, the pp-MOCVD method is single-step and produces adherent, conformal films with hierarchical porosity 
as evidenced in SEM, Fig. 2, and TEM, Fig. 6, analyses.

Future work includes determining the detailed crystallography of the anatase dendrites and their mechanism of growth in the pp-MOCVD process. Determining the location of rutile in the composite films will require extended TEM analysis. The nature and location of carbon in the films is of interest due its potential effects on adsorption of organic molecules, wettability and light absorption. It is also possible that free carbon has a synergistic effect on growth of this particular anatase morphology.

\section{Conclusions}

We report the evolution of the microstructure of titania films grown by pp-MOCVD from TTIP on fused silica substrates at $525^{\circ} \mathrm{C}$. The only variable in this study was the deposition time, which in pp-MOCVD relates to the number of pulses. The materials were characterised and PCA performance assessed. Nucleation was not studied, but three growth regimes were identified with early stage (10 pulse-40 pulse), transition stage (70 pulse- 80 pulse) and late stage (90 pulse-300 pulse) growth. Anatase and rutile were identified in all samples with thicknesses ranging from $101 \mathrm{~nm}$ to $4.0 \mu \mathrm{m}$. A strong anatase (220) texture and rutile (200) texture developed during transition stage and was maximal in late stage films. In early stage growth, films appear rough with rounded surface morphology. They have low RMS roughness, no detectable carbon, low texture coefficients and a linear growth rate $\left(37 \mathrm{~nm} \mathrm{~min}^{-1}\right)$. The transition stage films are columnar, and anatase starts to exhibit a dendritic morphology with secondary plates. They have increased RMS roughness, amorphous carbon, increasing texture coefficients and a higher growth rate than early stage films. Late stage films have large anatase dendrites growing along [110] perpendicular to the substrate and are highly textured in both anatase and rutile. They have the largest RMS roughness of the set, amorphous carbon and the highest average growth rate of $130 \mathrm{~nm} \mathrm{~min}^{-1}$.

The photocatalytic activity was highest in the films with highest surface roughness. The best performing film $(1.01 \mu \mathrm{m}$ thick $)$ had FQE of $(6.62 \pm 0.64) \times 10^{-4}$ molecules/photon and was 59 times higher than the commercial standard Pilkington Activ ${ }^{\mathrm{TM}}$. The surface roughness is associated with the highly branched anatase dendrites with secondary dendrite plate-like structures with dimensions of tens of $\mathrm{nm}$ in late stage growth. We postulate that the high PCA resulted from anatase/rutile polymorphic junctions, which 
decrease the recombination rate of photogenerated carriers; the nanoscale size of the two phases, which increases the surface area for reaction and increases the regions affected by electric fields at interdendritic and interphase interfaces; and the amorphous carbon phase, which promotes adsorption of organic molecules.

The growth conditions that produce this particular morphology of anatase with dispersed rutile and amorphous carbon are the subject of future work. Additionally, longer deposition times may yield a decrease or a stagnation of PCA depending on whether rutile eventually dominates the upper region of the films or whether a stagnation RMS roughness is obtained. It is important to understand the growth stages for each set of deposition parameters (temperature, pulse exposure, substrate) as part of overall process engineering and scale-up to achieve the desired coating with optimal properties.

\section{Acknowledgements}

The authors would like to thank Dr. Darryl Lee for help in sample preparation and Mike Flaws from University of Canterbury and Richard Easingwood from OCEM, University of Otago for their invaluable help in SEM and TEM analysis.

This work was supported by New Zealand Ministry for Business, Innovation and Employment under the High Value Manufacturing Science and Technology Research Program Contract No. CONT-42986-HVMSTR-UOC (UOCX1501) and a research contract from KOTI Technologies Limited.

\section{References}

[1] M. J. Lawrence, A. Kolodziej, P. Rodriguez, Controllable synthesis of nanostructured metal oxide and oxyhydroxide materials via electrochemical methods, Current Opinion in Electrochemistry 10 (2018) 7-15. doi:10.1016/j.coelec.2018.03.014.

[2] A. J. Gardecka, C. Bishop, D. Lee, S. Corby, I. P. Parkin, A. Kafizas, S. Krumdieck, High efficiency water splitting photoanodes composed of nano-structured anataserutile TiO2 heterojunctions by pulsed-pressure MOCVD, Applied Catalysis B: Environmental 224 (2018) 904-911. doi:10.1016/j.apcatb.2017.11.033.

[3] A. J. Gardecka, M. Lübke, C. F. Armer, D. Ning, M. V. Reddy, A. S. Williams, A. Lowe, Z. Liu, I. P. Parkin, J. A. Darr, Nb-doped rutile titanium dioxide nanorods 
for lithium-ion batteries, Solid State Sciences 83 (2018) 115-121. doi:10.1016/j. solidstatesciences.2018.07.004.

[4] J. Tian, Z. Zhao, A. Kumar, R. I. Boughton, H. Liu, Recent progress in design, synthesis, and applications of one-dimensional TiO2 nanostructured surface heterostructures: A review, Chemical Society Reviews 43 (20) (2014) 6920-6937. doi:10.1039/C4CS00180J.

[5] C. W. Dunnill, K. Page, Z. A. Aiken, S. Noimark, G. Hyett, A. Kafizas, J. Pratten, M. Wilson, I. P. Parkin, Nanoparticulate silver coated-titania thin films-Photooxidative destruction of stearic acid under different light sources and antimicrobial effects under hospital lighting conditions, Journal of Photochemistry and Photobiology A: Chemistry 220 (2) (2011) 113-123. doi:10.1016/j.jphotochem.2011.04.001.

[6] T. Luttrell, S. Halpegamage, J. Tao, A. Kramer, E. Sutter, M. Batzill, Why is anatase a better photocatalyst than rutile? - Model studies on epitaxial $\mathrm{TiO}_{2}$ films, Scientific Reports 4 (2014) 4043. doi:10.1038/srep04043.

[7] I. S. Cho, Z. Chen, A. J. Forman, D. R. Kim, P. M. Rao, T. F. Jaramillo, X. Zheng, Branched TiO2 Nanorods for Photoelectrochemical Hydrogen Production, Nano Letters 11 (11) (2011) 4978-4984. doi:10.1021/nl2029392.

[8] Z. Liu, X. Zhang, S. Nishimoto, M. Jin, D. A. Tryk, T. Murakami, A. Fujishima, Anatase TiO2 Nanoparticles on Rutile TiO2 Nanorods: A Heterogeneous Nanostructure via Layer-by-Layer Assembly, Langmuir 23 (22) (2007) 10916-10919. doi:10.1021/la7018023.

[9] D. O. Scanlon, C. W. Dunnill, J. Buckeridge, S. A. Shevlin, A. J. Logsdail, S. M. Woodley, C. R. A. Catlow, M. J. Powell, R. G. Palgrave, I. P. Parkin, G. W. Watson, T. W. Keal, P. Sherwood, A. Walsh, A. A. Sokol, Band alignment of rutile and anatase $\mathrm{TiO}_{2}$, Nature Materials 12 (9) (2013) 798-801. doi:10.1038/nmat3697.

[10] Y. Takahashi, H. Suzuki, M. Nasu, Rutile growth at the surface of TiO2 films deposited by vapour-phase decomposition of isopropyl titanate, Journal of the Chemical Society, Faraday Transactions 1: Physical Chemistry in Condensed Phases 81 (12) (1985) 3117-3125. doi:10.1039/F19858103117. 
[11] A. Goossens, E.-L. Maloney, J. Schoonman, Gas-Phase Synthesis of Nanostructured Anatase TiO2, Chemical Vapor Deposition 4 (3) (1998) 109-114. doi:10.1002/ (SICI) 1521-3862(199805) 04:03<109: :AID-CVDE109>3.0.C0;2-U.

[12] V. Siriwongrungson, S. Krumdieck, M. Alkaisi, Conformality Investigation of Titanium Dioxide Thin Films on 3-D Micrometer- and Nanometer-scale Features by Pulsed-Pressure Metal-organic CVD -, Chemical Vapor Deposition 17 (10-12) (2011) 327-336.

[13] Krumdieck S., Miya S. S., Lee D., Davies-Talwar S., Bishop C. M., Titania-based photocatalytic coatings on stainless steel hospital fixtures, physica status solidi c 12 (7) (2015) 1028-1035. doi:10.1002/pssc.201510040.

[14] S. Krumdieck, R. Gorthy, A. J. Gardecka, D. Lee, S. S. Miya, S. D. Talwar, M. I. J. Polson, C. Bishop, Characterization of photocatalytic, wetting and optical properties of $\mathrm{TiO} 2$ thin films and demonstration of uniform coating on a 3-D surface in the mass transport controlled regime, Surface and Coatings Technology 326 (2017) 402-410. doi:10.1016/j.surfcoat.2016.11.064.

[15] S. P. Krumdieck, R. Boichot, R. Gorthy, J. G. Land, S. Lay, A. J. Gardecka, M. I. J. Polson, A. Wasa, J. E. Aitken, J. A. Heinemann, G. Renou, G. Berthomé, F. Charlot, T. Encinas, M. Braccini, C. M. Bishop, Nanostructured TiO 2 anatase-rutile-carbon solid coating with visible light antimicrobial activity, Scientific Reports 9 (1) (2019) 1883. doi:10.1038/s41598-018-38291-y.

[16] K. L. Siefering, G. L. Griffin, Kinetics of Low-Pressure Chemical Vapor Deposition of TiO2 from Titanium Tetraisopropoxide, Journal of The Electrochemical Society 137 (3) (1990) 814-818. doi:10.1149/1.2086561.

[17] C.-A. Chen, Y.-M. Chen, Y.-S. Huang, D.-S. Tsai, K.-K. Tiong, P.-C. Liao, Synthesis and characterization of well-aligned anatase TiO2nanocrystals on fused silicavia metal-organic vapor deposition, CrystEngComm 11 (11) (2009) 2313-2318. doi:10.1039/B909433D.

[18] C. A. Chen, Y. M. Chen, A. Korotcov, Y. S. Huang, D. S. Tsai, K. K. Tiong, Growth and characterization of well-aligned densely-packed rutile $\mathrm{TiO} 2$ nanocrystals on 
sapphire substrates via metal-organic chemical vapor deposition, Nanotechnology 19 (7) (2008) 075611. doi:10.1088/0957-4484/19/7/075611.

[19] D. Lee, S. Krumdieck, S. D. Talwar, Scale-up design for industrial development of a PP-MOCVD coating system, Surface and Coatings Technology 230 (2013) 39-45. doi:10.1016/j.surf coat.2013.06.064.

[20] M. Klinger, A. Jäger, Crystallographic Tool Box (CrysTBox): Automated tools for transmission electron microscopists and crystallographers, Journal of Applied Crystallography 48 (Pt 6) (2015) 2012-2018. doi:10.1107/S1600576715017252.

[21] D. Nečas, P. Klapetek, Gwyddion: An open-source software for SPM data analysis, Open Physics 10 (1) (2011) 181-188. doi:10.2478/s11534-011-0096-2.

[22] A. Mills, J. Wang, Simultaneous monitoring of the destruction of stearic acid and generation of carbon dioxide by self-cleaning semiconductor photocatalytic films, Journal of Photochemistry and Photobiology A: Chemistry 182 (2) (2006) 181-186. doi:10.1016/j.jphotochem.2006.02.010.

[23] S.-C. Jung, Photocatalytic activities and specific surface area of TiO2 films prepared by CVD and sol-gel method, Korean Journal of Chemical Engineering 25 (2) (2008) 364-367. doi:10.1007/s11814-008-0060-z.

[24] S. Karim, M. E. Toimil-Molares, F. Maurer, G. Miehe, W. Ensinger, J. Liu, T. W. Cornelius, R. Neumann, Synthesis of gold nanowires with controlled crystallographic characteristics, Applied Physics A 84 (4) (2006) 403-407. doi:10.1007/ s00339-006-3645-6.

[25] C. J. Howard, T. M. Sabine, F. Dickson, Structural and thermal parameters for rutile and anatase, Acta Crystallographica Section B: Structural Science 47 (4) (1991) 462468. doi:10.1107/S010876819100335X.

[26] P. K. Chu, L. Li, Characterization of amorphous and nanocrystalline carbon films, Materials Chemistry and Physics 96 (2-3) (2006) 253-277. doi:10.1016/ j.matchemphys.2005.07.048. 
[27] W.-Q. Wu, H.-L. Feng, H.-S. Rao, Y.-F. Xu, D.-B. Kuang, C.-Y. Su, Maximizing omnidirectional light harvesting in metal oxide hyperbranched array architectures, Nature Communications 5 (2014) 3968. doi:10.1038/ncomms4968.

[28] A. Mills, A. Lepre, N. Elliott, S. Bhopal, I. P. Parkin, S. A. O’Neill, Characterisation of the photocatalyst Pilkington Activ $^{\mathrm{TM}}$ : A reference film photocatalyst?, Journal of Photochemistry and Photobiology A: Chemistry 160 (3) (2003) 213-224. doi: $10.1016 / \mathrm{S} 1010-6030(03) 00205-3$

[29] R. Quesada-Cabrera, C. Sotelo-Vázquez, M. Quesada-González, E. P. Melián, N. Chadwick, I. P. Parkin, On the apparent visible-light and enhanced UV-light photocatalytic activity of nitrogen-doped $\mathrm{TiO} 2$ thin films, Journal of Photochemistry and Photobiology A: Chemistry 333 (2017) 49-55. doi:10.1016/j.jphotochem. 2016.10 .013

[30] N. J. Platt, K. M. Kaye, G. J. Limburn, S. D. Cosham, A. N. Kulak, R. G. Palgrave, G. Hyett, Order of magnitude increase in photocatalytic rate for hierarchically porous anatase thin films synthesized from zinc titanate coatings, Dalton Transactions 46 (6) (2017) 1975-1985. doi:10.1039/C6DT04431J.

[31] E. Mirica, G. Kowach, H. Du, Modified Structure Zone Model to Describe the Morphological Evolution of ZnO Thin Films Deposited by Reactive Sputtering, Crystal Growth \& Design 4 (1) (2004) 157-159. doi:10.1021/cg025596b.

[32] C. V. Thompson, R. Carel, Texture development in polycrystalline thin films, Materials Science and Engineering: B 32 (3) (1995) 211-219. doi:10.1016/0921-5107(95) 03011-5.

[33] T. Karabacak, Thin-film growth dynamics with shadowing and re-emission effects, Journal of Nanophotonics 5 (1) (2011) 052501. doi:10.1117/1.3543822.

[34] R. A. L. Almeida, S. O. Ferreira, I. Ferraz, T. J. Oliveira, Initial pseudo-steady state \& asymptotic KPZ universality in semiconductor on polymer deposition, Scientific Reports 7 (1) (2017) 3773. doi:10.1038/s41598-017-03843-1.

[35] R. L. Penn, J. F. Banfield, Oriented attachment and growth, twinning, polytypism, 
and formation of metastable phases; insights from nanocrystalline $\mathrm{TiO} 2$, American Mineralogist 83 (9-10) (1998) 1077-1082. doi:10.2138/am-1998-9-1016.

[36] R. L. Penn, J. F. Banfield, Formation of rutile nuclei at anatase (112) twin interfaces and the phase transformation mechanism in nanocrystalline titania, American Mineralogist 84 (5-6) (1999) 871-876. doi:10.2138/am-1999-5-621.

[37] S.-C. Zhu, S.-H. Xie, Z.-P. Liu, Nature of Rutile Nuclei in Anatase-to-Rutile Phase Transition, Journal of the American Chemical Society 137 (35) (2015) 11532-11539. doi:10.1021/jacs.5b07734.

[38] F. L. Forgerini, R. Marchiori, A brief review of mathematical models of thin film growth and surfaces, Biomatter 4. doi:10.4161/biom.28871.

[39] S.-C. Jung, S.-J. Kim, N. Imaishi, Y.-I. Cho, Effect of TiO2 thin film thickness and specific surface area by low-pressure metal-organic chemical vapor deposition on photocatalytic activities, Applied Catalysis B: Environmental 55 (4) (2005) 253257. doi:10.1016/j.apcatb.2004.08.009.

[40] S.-C. Jung, N. Imaishi, Preparation, crystal structure, and photocatalytic activity of TiO2 films by chemical vapor deposition, Korean Journal of Chemical Engineering 18 (6) (2001) 867-872. doi:10.1007/BF02705610.

[41] L. Li, P. A. Salvador, G. S. Rohrer, Photocatalysts with internal electric fields, Nanoscale 6 (1) (2014) 24-42. doi:10.1039/C3NR03998F.

[42] K. W. Powers, M. Palazuelos, B. M. Moudgil, S. M. Roberts, Characterization of the size, shape, and state of dispersion of nanoparticles for toxicological studies, Nanotoxicology 1 (1) (2007) 42-51. doi:10.1080/17435390701314902.

[43] R. Leary, A. Westwood, Carbonaceous nanomaterials for the enhancement of TiO2 photocatalysis, Carbon 49 (3) (2011) 741-772. doi:10.1016/j.carbon.2010.10. 010 .

[44] N. M. Nursam, X. Wang, R. A. Caruso, Macro-/mesoporous titania thin films: Analysing the effect of pore architecture on photocatalytic activity using highthroughput screening, Journal of Materials Chemistry A 3 (48) (2015) 24557-24567. doi:10.1039/C5TA08959J. 
Table 1: Data summary for thin $\mathrm{TiO}_{2}$ films deposited by pp-MOCVD on fused silica substrates at $525^{\circ} \mathrm{C}$. Errors are standard deviation of measurements.

\begin{tabular}{|c|c|c|c|c|c|c|c|c|c|c|}
\hline $\begin{array}{c}\text { Pulses } \\
{[-]}\end{array}$ & \multicolumn{3}{|c|}{$\begin{array}{l}\text { Thickness } \\
\text { [nm] }\end{array}$} & \multirow{2}{*}{$\begin{array}{c}\text { Area ratio } \\
{[-]} \\
1.3\end{array}$} & \multirow{2}{*}{$\begin{array}{c}\text { RMS } \\
{[\mathrm{nm}]} \\
2\end{array}$} & \multirow{2}{*}{$\begin{array}{c}\text { TC A } 220 \\
{[-]} \\
2.0\end{array}$} & \multirow{2}{*}{$\begin{array}{c}\text { TC R200 } \\
{[-]} \\
1.0\end{array}$} & \multicolumn{3}{|c|}{$\begin{array}{c}\text { FQE } \\
{\left[10^{-4} \text { molecules/photon }\right]}\end{array}$} \\
\hline 10 & 101 & \pm & 8 & & & & & 0.12 & \pm & 0.10 \\
\hline 20 & 143 & \pm & 12 & 1.1 & 4 & 2.1 & 2.2 & 1.00 & \pm & 0.44 \\
\hline 30 & 176 & \pm & 7 & 1.2 & 4 & 2.2 & 2.5 & 0.69 & \pm & 0.28 \\
\hline 40 & 320 & \pm & 12 & 1.4 & 14 & 2.0 & 2.2 & 0.36 & \pm & 0.21 \\
\hline 70 & 494 & \pm & 14 & 1.2 & 10 & 3.6 & 2.9 & 0.33 & \pm & 0.19 \\
\hline 80 & 452 & \pm & 26 & 1.4 & 13 & 3.3 & 3.0 & 1.18 & \pm & 0.48 \\
\hline 90 & 519 & \pm & 30 & 1.4 & 13 & 3.1 & 3.0 & 0.53 & \pm & 0.19 \\
\hline 120 & 725 & \pm & 11 & 1.7 & 18 & 2.8 & 3.0 & 0.28 & \pm & 0.05 \\
\hline 200 & 1067 & \pm & 16 & 1.5 & 40 & 3.5 & 3.0 & 6.62 & \pm & 0.64 \\
\hline 300 & 4007 & \pm & 73 & 1.7 & 62 & 4.5 & 2.5 & 5.84 & \pm & 1.09 \\
\hline
\end{tabular}

$\mathrm{RMS}=$ root mean square roughness. $\mathrm{TC}$ A220 $=$ texture coefficient for anatase 220 reflection with $N=5$. TC R200 = texture coefficient for rutile 200 reflection with $N=3$. 

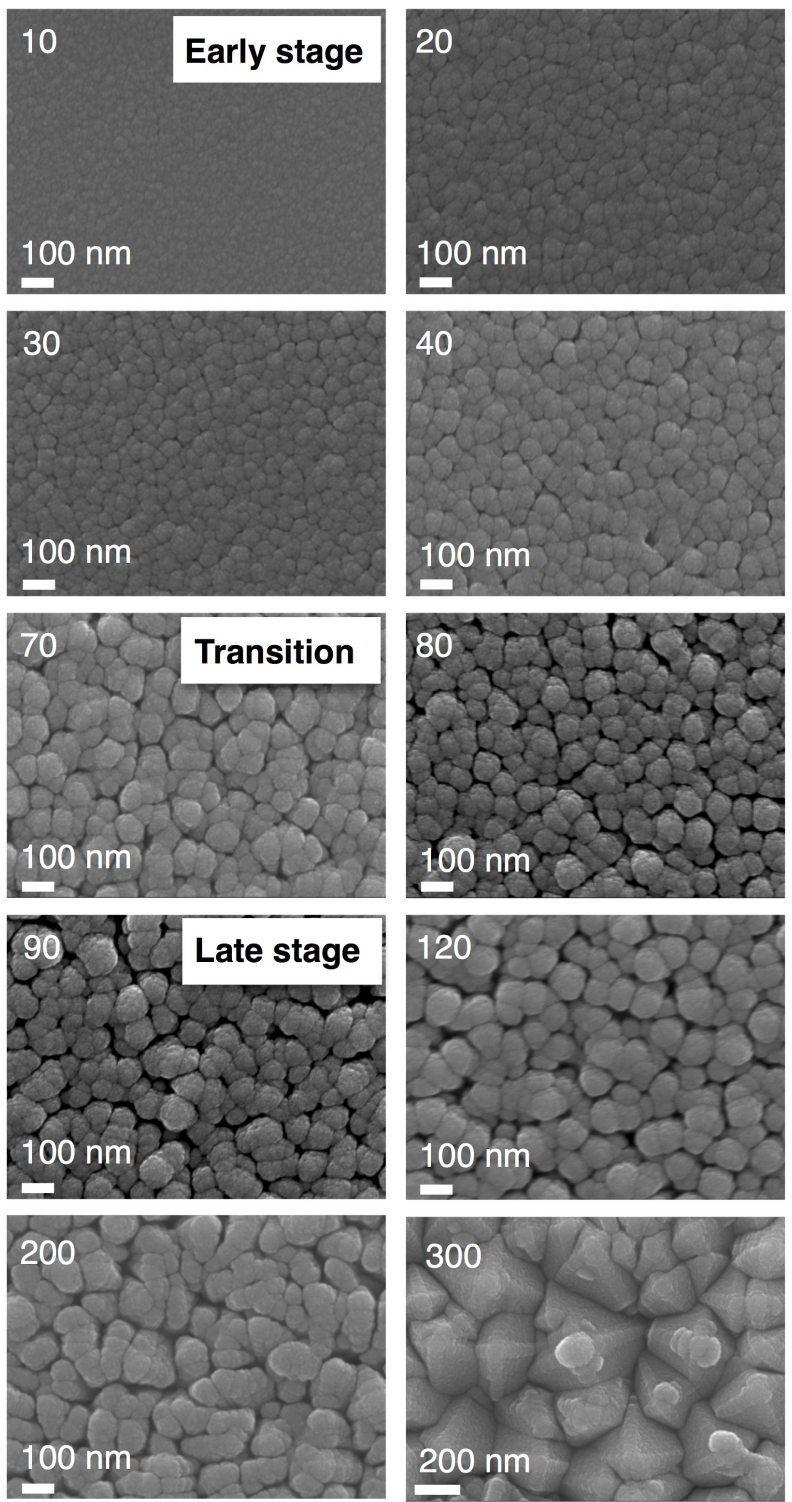

Figure 1: SEM plan view images of films deposited with 10 pulses-300 pulses. Growth stages are identified: early stage 10 pulses -40 pulses, transition stage 70 pulses- 80 pulses and late stage 90 pulses-300 pulses. 


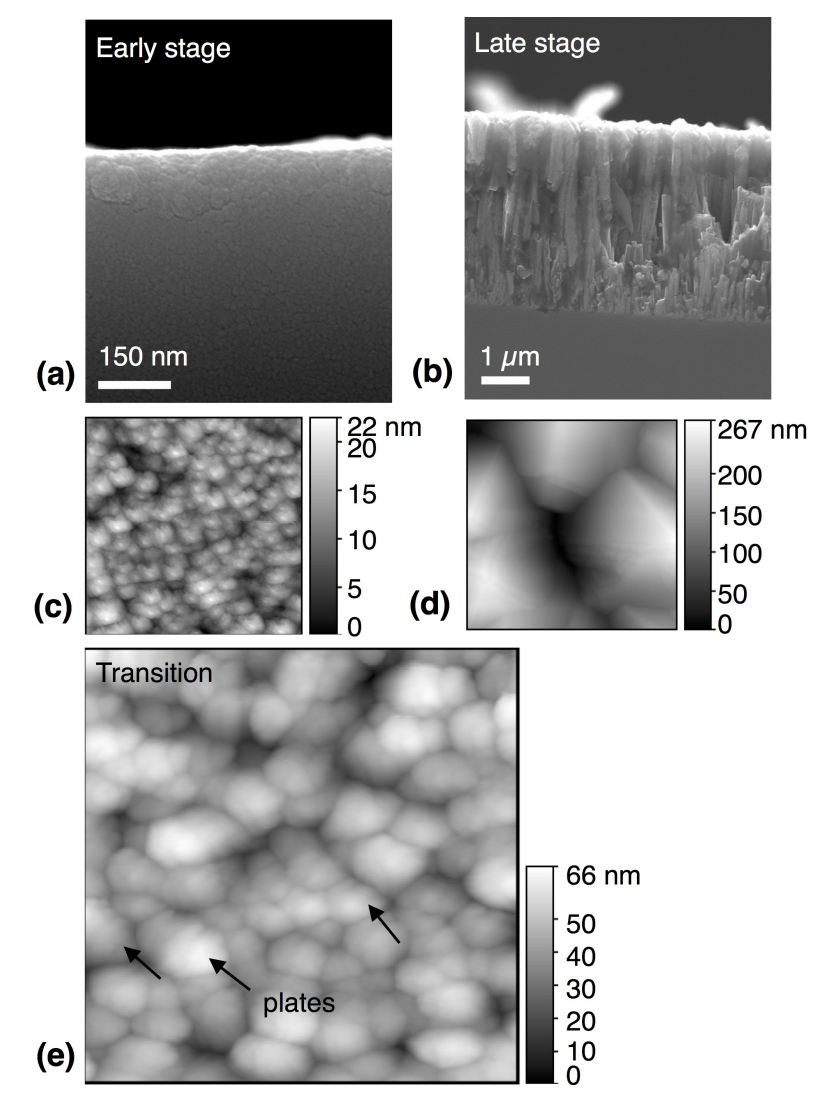

Figure 2: Morphology of early, transition and late stage films. SEM cross sections of (a) 20 pulse and (b) 300 pulse films with corresponding AFM contour maps of (c) 20 pulse and (d) 300 pulse films. The AFM contour map of 80 pulse film (e) shows secondary plate formation (arrows) during transition stage growth. AFM maps are $500 \mathrm{~nm} \times 500 \mathrm{~nm}$ regions of each sample. 


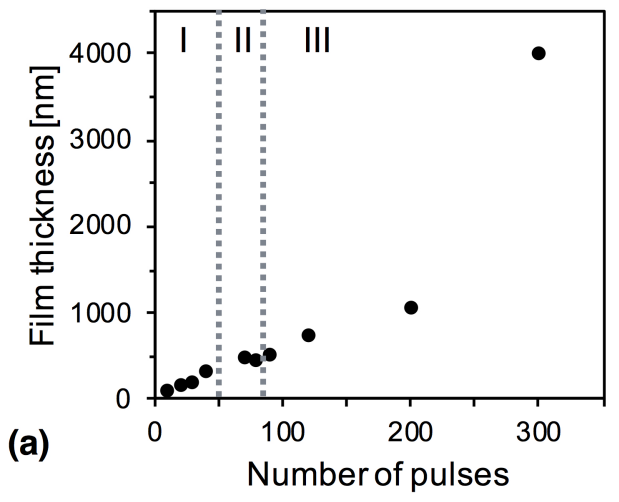

(b)
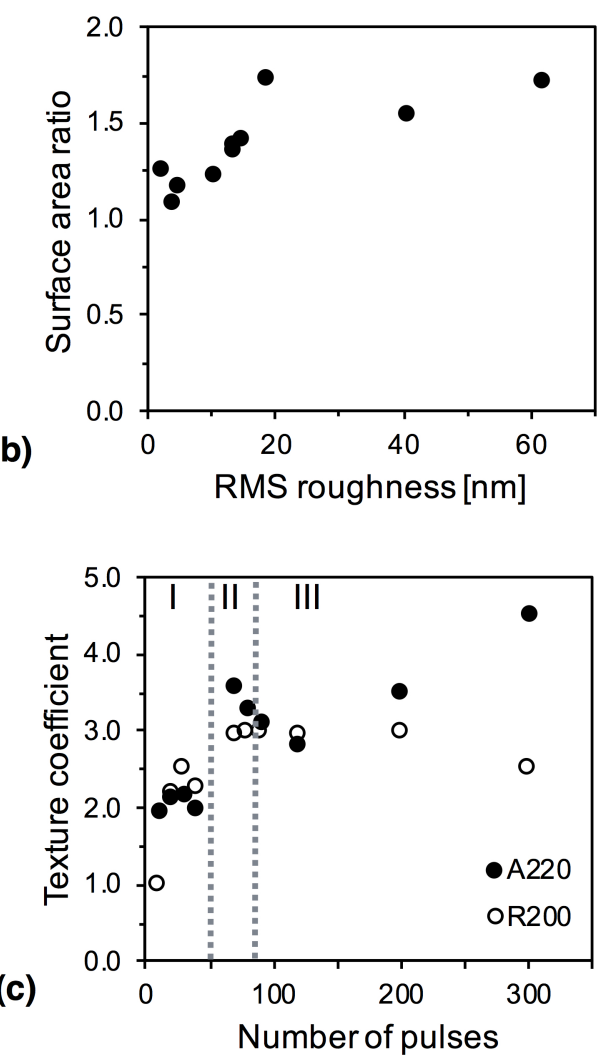

Figure 3: Microstructural measures of $\mathrm{TiO}_{2}$ films with (a) film thickness against number of pp-MOCVD pulses, (b) surface area ratio (total surface area / projected surface area) against RMS roughness from AFM and (c) texture coefficients A220 for anatase (220) reflections with $\mathrm{N}=5$ and $\mathrm{R} 200$ for rutile (200) reflections with $\mathrm{N}=3$ against number of pp-MOCVD pulses. Error bars for film thickness lie within the markers. The relative error in the A220 texture coefficient was up to $250 \%$ in early stage films, but less than $5 \%$ for transition and late stage films with $>70$ pulses. Growth stages are identified: I $=$ early, II = transition and III = late. 

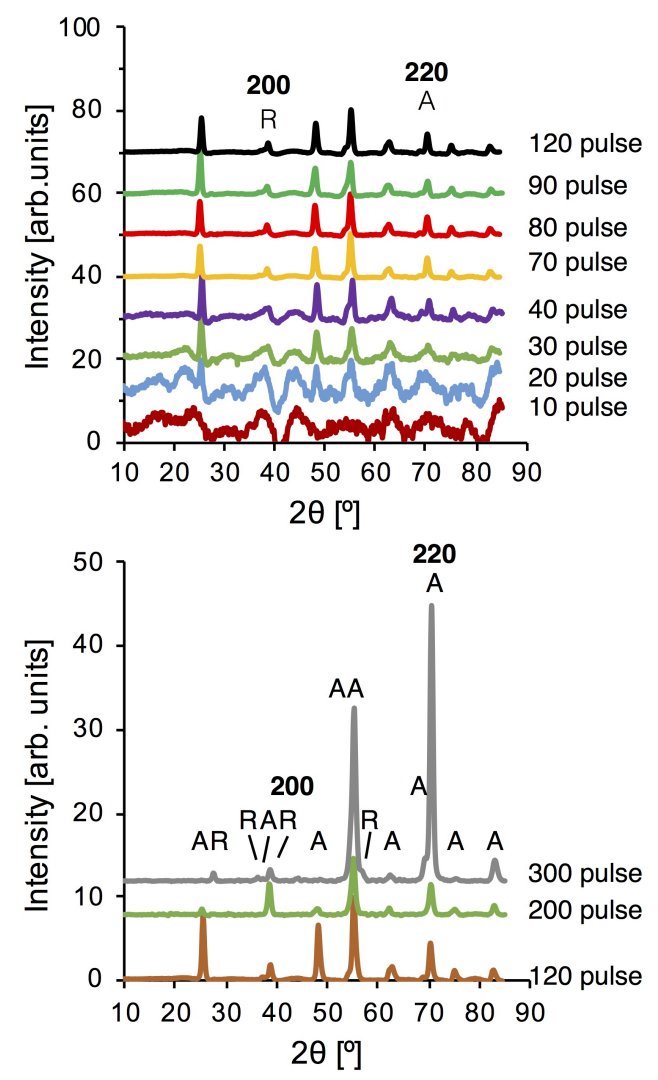

Figure 4: XRD patterns for all films with $\mathrm{A}=$ anatase and $\mathrm{R}=$ rutile reflections labelled. 


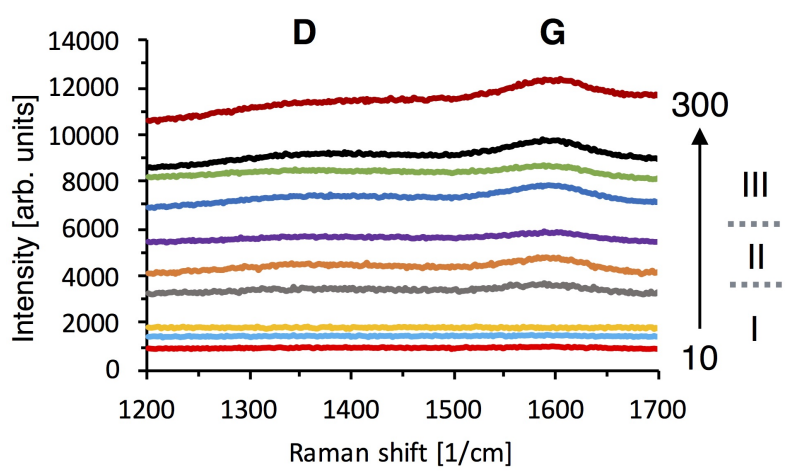

Figure 5: Raman spectra for all films in the range of interest for carbon D and G peaks. Carbon is clearly identified in films $>30$ pulses. Growth stages are identified: I = early, $\mathrm{II}=$ transition and III = late. 
(a)
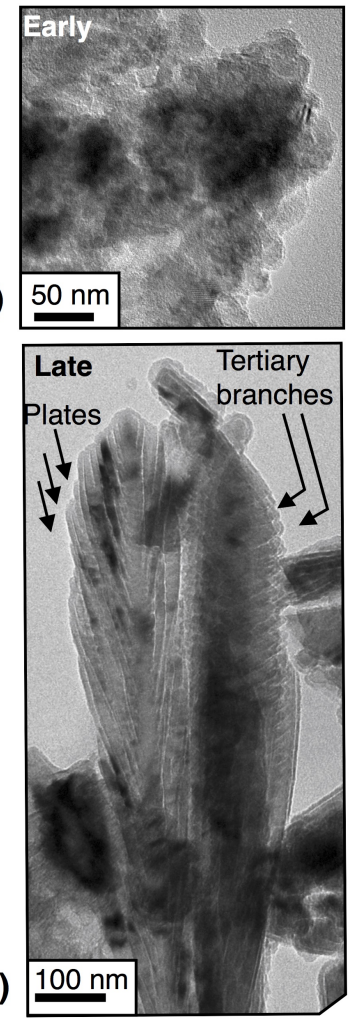

(b)

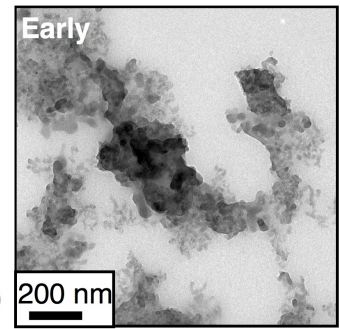

(d)
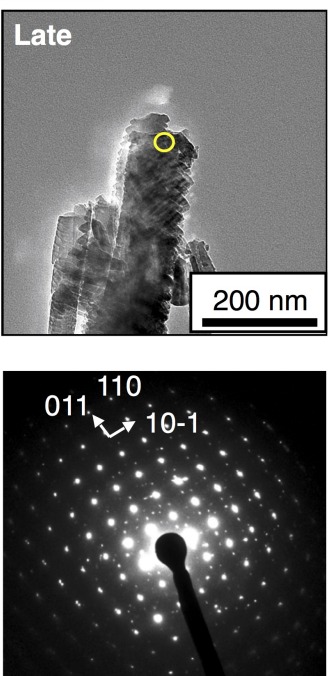

Figure 6: TEM analysis of material from titania thin films from early stage (a-b) and late stage growth (c-e). (a) small clusters from $140 \mathrm{~nm}$ film (20 pulses), (b) larger clusters from $176 \mathrm{~nm}$ film (30 pulses), (c) anatase dendrites showing nanoscale structure from $1.1 \mu \mathrm{m}$ film (200 pulses) with secondary plates and tertiary branches labelled in the two adjacent dendrites, (d) fragments of anatase dendrites from $1.1 \mu \mathrm{m}$ film (200 pulses) showing secondary dendrite plates and (e) SAED pattern from area in circle in (d) showing [110] growth direction of dendrites. 


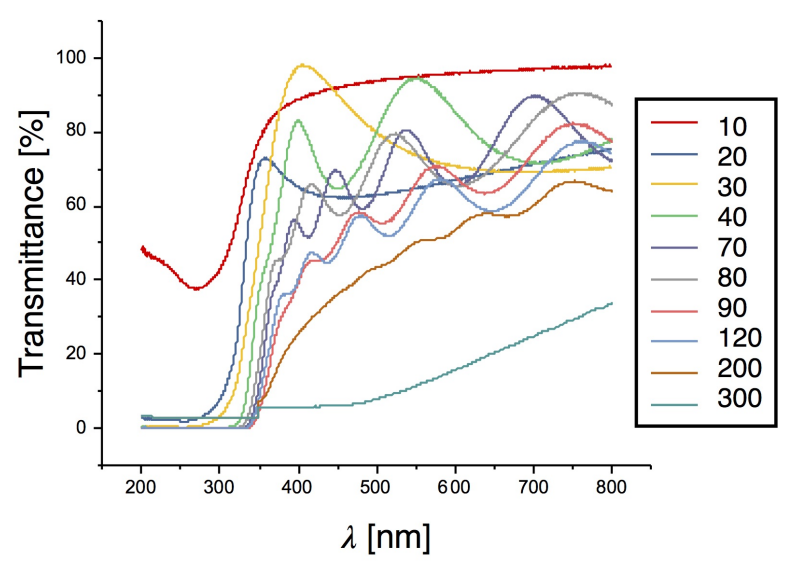

Figure 7: Transmission UV-vis spectra showing gradual decrease of transmittance with increasing number of growth pulses from films of 10 pulses-300 pulses. 


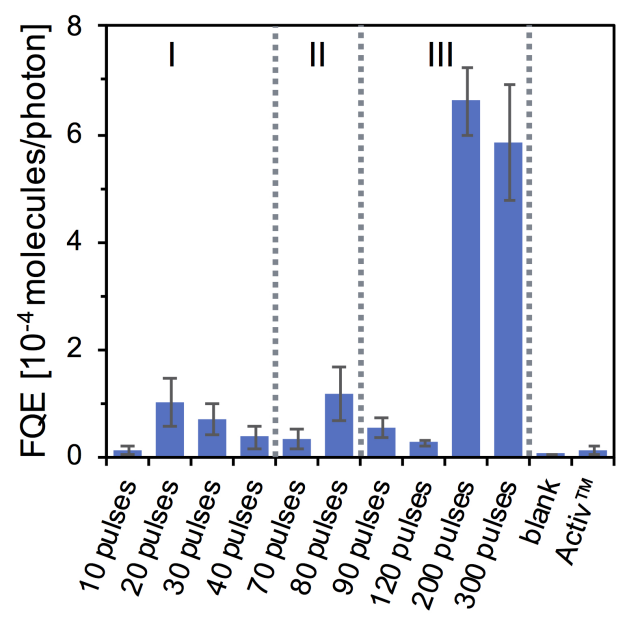

Figure 8: Formal quantum efficiency obtained by degradation of stearic acid under UVA irradiation. Late stage films have highest performance. Growth stages are identified: I $=$ early, $\mathrm{II}=$ transition and III $=$ late. 


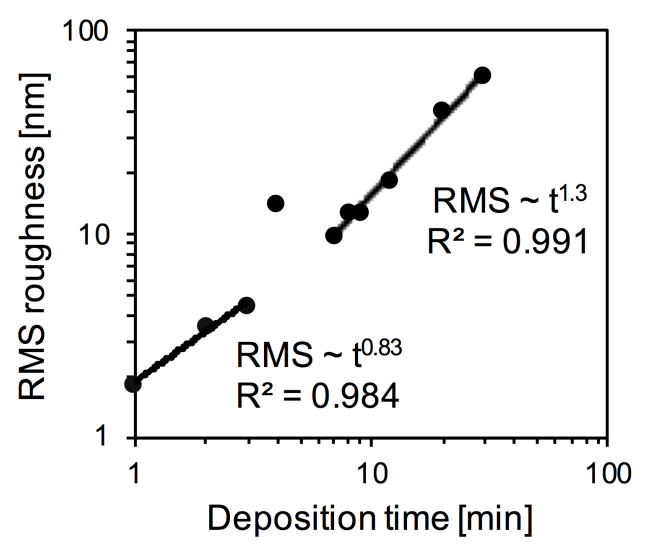

Figure 9: Dynamic scaling analysis of interface width (RMS roughness) with growth time showing two regimes. The 40 pulse sample is an outlier here. 


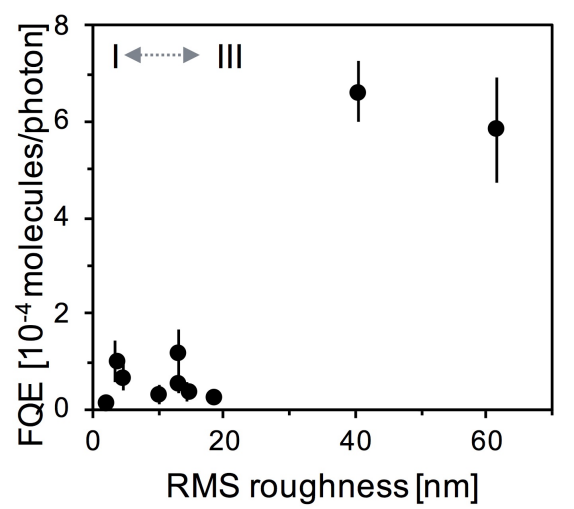

Figure 10: Relationship between FQE and RMS roughness for titania films showing that the late stage films have significantly higher PCA. Growth stages are identified: I = early and III = late. 